\title{
Evaluation of Fasting Blood Sugar and Lipid Profile in Patients with Type 1 and Type 2 Diabetes and Normoglycemic Individuals in Gorgan, Northeastern Iran
}

\author{
Nasim Arab Sarhadi', Mohammad-ebrahim Fakhreddin-nejad ${ }^{1}$, Mohammad-hassan Rajabi ${ }^{1}$, \\ Soleiman Mokarrari ${ }^{1}$, Ebrahim Naghipour ${ }^{1}$, Hooshmand Karkon ${ }^{1}$, Mohammad Mostakhdem \\ Hashemi*2 \\ 1. Shohada Hospital, Gonbad-e-Kavoos, Golestan, Iran \\ 2. Metabolic Disorders Research Center, Golestan University of Medical Sciences, Gorgan, Iran
}

\begin{abstract}
Background and objectives: Diabetes mellitus is a major public health issue worldwide. Poorly managed type 1 and type 2 diabetics are at risk of developing several complications, including cardiovascular disease. Therefore, monitoring lipid profile and glycemic control in such patients are of great importance for reducing the risk of developing diabetic complications. In this study, we measured the level of fasting blood sugar (FBS) and lipid profile components in patients with either type 1 or type 2 diabetes and non-diabetic individuals.

Methods: This cross-sectional case-control study was conducted on 50 type 1 diabetics, 50 type 2 diabetics and 50 normoglycemic individuals (controls). All subjects were matched in terms of age and gender. Serum level of FBS, total cholesterol (TC), triglyceride (TG), low-density lipoprotein (LDL) and high-density lipoprotein (HDL) was measured using commercial kits. Statistical analysis was performed using SPSS software (version 16) at significance level of 0.05 .

Results: The levels of FBS, TC, TG, LDL and HDL were higher in both case groups compared to the controls. The value of these variables (except for TC) was significantly higher in patients with type 1 diabetes than in patients with type 2 diabetes.

Conclusion: Our results show that patients with type 2 diabetes have lower levels of HDL compared to T1DM patients, which might increase the risk of developing cardiovascular complications in these patients.

Keywords: Cardiovascular Diseases; Diabetes Mellitus, Type 1; Diabetes Mellitus, Type 2; Lipid profile; FBS
\end{abstract}

Received: 2019/04/05

Revised: 2019/04/15

Published: 2019/04/29

*Correspondence: Mohammad Mostakhdem Hashemi, Department of Biochemistry and Biophysics, Faculty of Medicine, Falsafi campus of Golestan University of Medical Sciences, Gorgan, Iran

Tel: +98-9141734551

Email: Mohammad_hashemi69@yahoo.com 


\section{INTRODUCTION}

Diabetes mellitus (DM) is a group of metabolic disorders characterized by hyperglycemia, which may be caused by impaired insulin secretion, insulin dysfunction or both $(1,2)$. The main subtypes of diabetes are type $1 \mathrm{DM}$ (T1DM) and type 2 DM (T2DM) (1) that affect almost 200 million people all around the world (3). Cardiovascular disease (CVD) is the main cause of death in diabetics, especially in T2DM patients (4). The American Heart Association (AHA) has emphasized the role of dyslipidemia and the importance of early diagnosis of cardiovascular risk factors in diabetics and patients with insulin resistance (5). The most prominent alterations of lipid fractions in diabetics mostly consist of lowdensity lipoprotein (LDL) elevation, decreased high-density lipoprotein (HDL) level or both (6-8). The elevated LDL can deposit in the arterial intima and result in hardening of blood vessels and arteries, a common complication of diabetes known as atherosclerosis. This condition may ultimately lead to arterial obstruction, blood clot formation and myocardial infarction (MI) or stroke in severe circumstances. Therefore, controlling dietary intake of lipids and serum levels of lipid fractions, particularly cholesterol is an important approach for prevention of diabetic complications $(9,10)$. In addition, elevation of triglyceride (TG) or triacylglycerole is another known risk factor for CVD (11). In this study, we measured and compared the level of fasting blood sugar (FBS) and lipid profile of patients with either type 1 or type 2 diabetes with that of nondiabetic individuals.

\section{MATERIALS AND METHODS Study population}

The present study was conducted on 50 T1DM patients, 50 T2DM patients and 50 normoglycemic individuals (controls). After explaining the study protocol and objectives, written consent was taken from all subjects. All subjects were matched in terms of age and gender. Diabetes subtype was approved by an endocrinologist. Exclusion criteria included unwillingness to continue participation in the study and a history of kidney or liver disease, operation, transplantation or malignancy.

\section{Sampling and biochemical assays}

Peripheral blood samples were collected into polystyrene tubes after a 10-hour fasting period. Serum of each sample was separated and stored at $-20{ }^{\circ} \mathrm{C}$ until biochemical testing. The level of FBS (Darman Faraz Kave, lot: 1022), total cholesterol (TC, Pars Azmun, lot: 94005), TG (Pars Azmun, lot: 94004) and HDL (Pars Azmun, lot: 93005) were measured by spectrophotometric method using commercially available kits. LDL level was calculated based on the Friedewald equation (12): $\mathrm{LDL}=\mathrm{TC}-\left(\mathrm{HDL}+\frac{\mathrm{TG}}{5}\right)$.

\section{Statistical analysis}

All statistical analyses were done using SPSS (version 16). Normality of data distribution was assessed using the Shapiro-Wilk test. Mean of each variable was compared using one-way ANOVA and Kruskal-Wallis test followed by Tukey's post-hoc test. Intragroup correlations were assessed using the Pearson's and Spearman's correlation coefficient in the case of parametric and non-parametric data, respectively. All analyses were performed at significance of 0.05 .

\section{RESULTS}

Mean age of T1DM, T2DM and control subjects was $48.4 \pm 18.5,54.4 \pm 12.86$ and $49.8 \pm 15.6$ years, respectively. FBS level was significantly higher in both diabetes groups compared to the control group but did not differ significantly between the type 1 and type 2 diabetics. HDL and TG levels were significantly higher in type 1 diabetics compared to the controls. In addition, the value of these variables was significantly higher in T1DM subjects than in T2DM subjects $(\mathrm{P}=0.045)$ (Table 1). However, HDL and TG levels did not differ significantly between the T2DM patients and healthy controls. 
Table 1. Levels (mean \pm standard deviation) of FBS and lipid profile components in the diabetes and control groups

\begin{tabular}{|c|c|c|c|c|}
\hline $\begin{array}{c}\text { Groups } \\
\text { Parameters }\end{array}$ & T1DM $(\mathbf{n}=50)$ & T2DM (n=50) & Control $(n=50)$ & P-value \\
\hline FBS (mg/dl) & $191.38 \pm 81.2$ & $181.73 \pm 66.82$ & $90.45 \pm 9.87$ & $\begin{array}{l}\mathrm{P}_{1}=0.854 \\
\mathrm{P}_{2}<0.001 \\
\mathrm{P}_{3}<0.001\end{array}$ \\
\hline TC (mg/dl) & $197.79 \pm 56.83$ & $204 \pm 155.93$ & $139.97 \pm 52.43$ & $\begin{array}{c}\mathrm{P}_{1}=0.248 \\
\mathrm{P}_{2}<0.001 \\
\mathrm{P}_{3}<0.01\end{array}$ \\
\hline TG (mg/dl) & $188.88 \pm 91.49$ & $151.15 \pm 111.01$ & $144.6 \pm 88.91$ & $\begin{array}{l}\mathrm{P}_{1}=0.036 \\
\mathrm{P}_{2}=0.029 \\
\mathrm{P}_{3}=0.443\end{array}$ \\
\hline HDL (mg/dl) & $52.96 \pm 16.16$ & $44.69 \pm 11.83$ & $39.32 \pm 12.58$ & $\begin{array}{c}\mathrm{P}_{1}=0.045 \\
\mathrm{P}_{2}<0.001 \\
\mathrm{P}_{3}=0.08\end{array}$ \\
\hline LDL (mg/dl) & $119.54 \pm 94.05$ & $104.27 \pm 50.23$ & $73.1 \pm 39.34$ & $\begin{array}{l}\mathrm{P}_{1}=0.741 \\
\mathrm{P}_{2}<0.001 \\
\mathrm{P}_{3}=0.006\end{array}$ \\
\hline
\end{tabular}

$\mathrm{P}_{1}$ : comparison between T1DM and T2DM patients.

$\mathrm{P}_{2}$ : comparison between T1DM and control subjects.

$\mathrm{P}_{3}$ : comparison between T2DM and control subjects.

In T1DM patients, there was a negative correlation between age and FBS $(\mathrm{P}=0.024$, $\mathrm{r}=-0.459)$. TG was directly correlated with TC $(\mathrm{P}=0.003, \quad \mathrm{r}=0.581)$ and LDL $(\mathrm{P}=0.005$, $\mathrm{r}=0.553)$. Duration of disease also showed a negative correlation with FBS level $(\mathrm{P}=0.043$, $\mathrm{r}=-0.416)$.

Similar to patients with T1DM, TC had a direct correlation with TG in T2DM patients $(\mathrm{P}=0.023, \mathrm{r}=0.443)$. Moreover, there was a positive correlation between HDL and LDL in type 2 diabetics $(\mathrm{P}=0.047, \mathrm{r}=0.392)$.

Based on the medical history of subjects, seven subjects with T2DM had a history of MI. Level of TC and FBS in these patients was significantly higher than in patients without a history of $\mathrm{MI}(\mathrm{P}=0.023$, data not shown). However, such analysis was not performed for type 1 diabetics due to lack of sufficient previous medical records.

\section{DISCUSSION}

We found significant variations in the level of lipid profile components between diabetics and non-diabetic individuals. Several factors are involved in the alteration of lipid profile following DM (13). The increased FBS level in diabetics promotes lipid peroxidation through stimulation of non-enzymatic glycation of proteins and formation of advanced glycated end-products (14). In such circumstances, the initiation of a vicious cycle consisting of apolipoproteins peroxidation and lipoproteins metabolism defects can aggravate this pathologic condition. Suryawanshi and colleagues observed a high level of lipid peroxides in diabetic patients with CVD complications (14). Similarly, we noted an increase in the lipid components such as LDL that are susceptible to peroxidation.

Interestingly, we found that T1DM patients had higher levels of HDL compared to controls, which is inconsistent with results of previous studies (15). Moreover, we observed that HDL level was significantly higher in T2DM patients with no history of statin therapy. Inconsistent with this finding, Marin et al. claimed that HDL level is higher in both obese and lean non-diabetic individuals compared to patients with T2DM who do not consume lipid-lowering drugs (16). In the present study, both HDL and LDL levels were significantly higher in patients with T1DM 
compared to controls. In such circumstances, the positive effect of high HDL may be masked by LDL elevation. However, we could not find a precise explanation for the concurrent elevation of LDL and HDL in T1DM patients.

In a study by Drexel et al., TG level was higher in T2DM patients compared to nondiabetic individuals (17). However, we observed this significant difference between T1DM patients and healthy controls. Nevertheless, this incongruity could be related to the fact that we had not investigated consumption of lipid-lowering medications or diet of subjects.

The levels of total cholesterol had a positive correlation with LDL and HDL levels in T1DM patients, while in T2DM patients, TG and LDL levels increased concurrently.

\section{CONCLUSION}

Both types of DM alter lipid profile components, which may increase the risk of developing cardiovascular complications in diabetics. Our results show that patients with T2DM have lower levels of HDL compared to T1DM patients, which might worsen the cardiovascular complications in these patients.

\section{ACKNOWLEDGMENTS}

The authors would like to express their appreciation to all participants in the study and staff of the Shohada hospital in Gonbade-Kavoos, Iran.

\section{DECLARATIONS}

\section{Funding}

This study was approved and supported by the Damghan Azad University (registration code: 14230520931011).

\section{Ethics approvals and consent to participate}

Written consent was obtained from all participants.

\section{Conflict of interest}

The authors declare that there is no conflict of interest regarding the publication of this article.

\section{REFERENCES}

1. World Health Organization. Definition, diagnosis and classification of diabetes mellitus and its complications: report of a WHO consultation. Part 1, Diagnosis and classification of diabetes mellitus. Geneva: World health organization; 1999.

2. Larsson SC, Wallin A, Håkansson N, Stackelberg O, Bäck M, Wolk A. Type 1 and type 2 diabetes mellitus and incidence of seven cardiovascular diseases. International journal of cardiology.

2018;262:66-70.

[DOI:10.1016/j.ijcard.2018.03.099]

3. Saaddine JB, Cadwell B, Gregg EW, Engelgau MM, Vinicor F, Imperatore G, Narayan KV. Improvements in diabetes processes of care and intermediate outcomes: United States, 1988-2002. Annals of internal medicine. 2006 ;144(7):465-74. [DOI:10.7326/0003-4819-144-7-20060404000005]

4. Verges B. Lipid modification in type 2 diabetes: the role of LDL and HDL. Fundamental \& clinical pharmacology. 2009;23(6):681-5. [DOI:10.1111/j.1472-8206.2009.00739.x]

5. Fox CS, Golden SH, Anderson C, Bray GA, Burke LE, De Boer IH, Deedwania P, Eckel RH, Ershow AG, Fradkin J, Inzucchi SE. Update on prevention of cardiovascular disease in adults with type 2 diabetes mellitus in light of recent evidence: a scientific statement from the American Heart Association and the American Diabetes Association. Circulation. 2015 Aug 25;132(8):691-718.

[DOI:10.1161/CIR.0000000000000230]

6. Pyörälä K, Laakso M, Uusitupa M. Diabetes and atherosclerosis: an epidemiologic view. Diabetes/metabolism reviews. 1987;3(2):463-524. [DOI:10.1002/dmr.5610030206]

7. Haffner SM, Lehto S, Rönnemaa T, Pyörälä K, Laakso M. Mortality from coronary heart disease in subjects with type 2 diabetes and in nondiabetic subjects with and without prior myocardial infarction. New England journal of medicine. 1998 ;339(4):229-34.

[DOI:10.1056/NEJM199807233390404] 
8. Turner RC, Millns H, Neil HA, Stratton IM, Manley SE, Matthews DR, Holman RR. Risk factors for coronary artery disease in non-insulin dependent diabetes mellitus: United Kingdom Prospective Diabetes Study (UKPDS: 23). Bmj. 1998;

[DOI:10.1136/bmj.316.7134.823] 316(7134):823-8.

9. Kim M, Kim M, Yoo HJ, Lee E, Chae JS, Lee $\mathrm{SH}$, Lee JH. A promoter variant of the APOA5 gene increases atherogenic LDL levels and arterial stiffness in hypertriglyceridemic patients. PloS one. $2017 \quad ; 12(12): e 0186693$. [DOI:10.1371/journal.pone.0186693]

10. Hashemi MM, Mansourian AR, Kosari E, Marjani A. Serum Levels of Nitric Oxide, Lipid Profiles, and Fasting Blood Glucose and Their Associations in Subclinical Hypothyroid Women Before and After Treatment by Levothyroxine. Transylvanian Review. 2016; 24 (10): 2689-2696.

11. Taskinen MR. Quantitative and qualitative lipoprotein abnormalities in diabetes mellitus. Diabetes. 1992; 41(Suppl. 2):12-17. [DOI:10.2337/diab.41.2.S12]

12. Mehta R, Reyes-Rodríguez E, Bello-Chavolla OY, Guerrero-Díaz AC, Vargas-Vázquez A, Cruz-Bautista I, Aguilar-Salinas CA. Performance of LDL-C calculated with Martin's formula compared to the Friedewald equation in familial combined hyperlipidemia. Atherosclerosis. 2018; 277:204-10.

[DOI:10.1016/j.atherosclerosis.2018.06.868]

13. Suckling KE, Jackson B. Animal models of human lipid metabolism. Progress in lipid research. 1993; 32(1):1-24. [DOI:10.1016/01637827(93)90002-E]

14. Suryawanshi NP, Bhutey AK, Nagdeote AN, Jadhav AA, Manoorkar GS. Study of lipid peroxide and lipid profile in diabetes mellitus. Indian Journal of Clinical Biochemistry. 2006; 21(1):126. [DOI:10.1007/BF02913080]

15. Rosenblit PD. Common medications used by patients with type 2 diabetes mellitus: what are their effects on the lipid profile?. Cardiovascular diabetology. 2016; 15(1):95. [DOI:10.1186/s12933-016-0412-7]
16. Marin MT, Dasari PS, Tryggestad JB, Aston CE, Teague AM, Short KR. Oxidized HDL and LDL in adolescents with type 2 diabetes compared to normal weight and obese peers. Journal of Diabetes and its Complications. 2015; 29(5):679-85.

[DOI:10.1016/j.jdiacomp.2015.03.015]

17. Drexel H, Aczel S, Marte T, Benzer W, Langer P, Moll W, Saely CH. Is atherosclerosis in diabetes and impaired fasting glucose driven by elevated LDL cholesterol or by decreased HDL cholesterol?. Diabetes care. 2005; 28(1):101-7. [DOI:10.2337/diacare.28.1.101] 